\title{
Cyclopamine increases the radiosensitivity of human pancreatic cancer cells by regulating the DNA repair signal pathway through an epidermal growth factor receptor-dependent pathway
}

\author{
XIAO-YANG WU ${ }^{1 *}, \mathrm{JUN} \mathrm{CHE}^{2 *}$, KE-KANG SUN ${ }^{1}$, XIAO-JUN SHEN ${ }^{1}$, \\ DONG YANG ${ }^{1}$, NING ZHONG ${ }^{1}$ and $\mathrm{HUA} \mathrm{ZHAO}^{3}$ \\ ${ }^{1}$ Department of Gastrointestinal Surgery, Division of Thoracic Surgery, Kunshan First People's Hospital \\ Affiliated to Jiangsu University, Kunshan, Jiangsu 215300; ${ }^{2}$ Department of Radiation Oncology, \\ Fourth Hospital of Wuxi City, Wuxi, Jiangsu 214062; ${ }^{3}$ Department of General Surgery, First Hospital \\ Affiliated to Soochow University, Suzhou, Jiangsu 215006, P.R. China
}

Received December 16, 2012; Accepted July 5, 2013

DOI: $10.3892 / \mathrm{mmr} .2013 .1605$

\begin{abstract}
Pancreatic cancer is an aggressive malignancy with a characteristic metastatic course of disease and resistance to conventional radiotherapy. As a result, the continual development of novel therapeutic agents is required to improve the current situation. In the present study, the effect of the hedgehog pathway inhibitor, cyclopamine, on cellular radiosensitivity was determined in K-RAS ${ }_{\mathrm{wt}}$ Colo-357 and K-RAS ${ }_{\mathrm{mt}}$ SW-1990 human pancreatic cancer cell lines using the clonogenic survival assay. Apoptosis and cell cycle distribution were detected using flow cytometry assay. Following irradiation (30 mins), residual double-strand breaks were quantified by identification of $\gamma$-H2AX foci of micronuclei and radiation-induced $\gamma-\mathrm{H} 2 \mathrm{AX}$, p-ATM, DNA-PKcs and Ku70 expression was analyzed using western blot analysis. The epidermal growth factor (EGF) and EGF receptor (EGFR) inhibitor, gefitinib, were utilized to determine the related mechanisms. The results revealed that cyclopamine treatment significantly reduced cell clonogenic survival but failed to induce apoptosis and radiation-induced G2 arrest. Flow cytometry revealed that cyclopamine treatment enhanced $\gamma$-H2AX foci in Colo-357 and SW-1990 cells exposed to irradiation. In addition, radiation-induced p-ATM, DNA-PKcs and Ku70 were all inhibited. EGF also rescued pancreatic cancer cells from cyclopamine-induced H2AX phosphorylation following irradiation. Thus, cyclopamine
\end{abstract}

Correspondence to: Professor Hua Zhao, Department of General Surgery, First Hospital Affiliated to Soochow University, Shizi Road, Suzhou, Jiangsu 215006, P.R. China

E-mail: fsyx7skk@163.com

${ }^{*}$ Contributed equally

Key words: cyclopamine, radiosensitivity, pancreatic cancer, epidermal growth factor receptor enhanced the radiosensitivity of human pancreatic cancer cells, in part, through an EGFR-dependent pathway, indicating a rational approach in combination with radiotherapy.

\section{Introduction}

Pancreatic cancer is a lethal disease that is notoriously difficult to treat (1). Only a small proportion of cases are curative through surgical resection and standard chemoradiotherapy for patients with advanced disease only has modest effects with substantial toxicity $(2,3)$. Clearly, the continual development of novel therapeutic agents is required to improve the current situation.

Several studies on biological approaches targeting the molecular abnormalities of pancreatic cancer are available (4-6). One such pathway is the hedgehog $(\mathrm{Hh})$ signaling pathway, which specifies patterns of cell growth and differentiation during embryogenesis in a wide range of tissues (7). In addition to its function in developmental patterning, the Hh pathway is also important in maintaining the homeostasis of mature tissues and the number of somatic cells in various organs. This pathway represents an attractive target for drug development and has shown promise in clinical trials of cancer treatments. The specificity of cyclopamine for the Hh pathway is demonstrated by the absence of cytotoxicity in cells that lack Hh signaling.

The K-ras oncogene mutation occurs in $75-90 \%$ of pancreatic cancers $(8,9)$. The gene encodes a $21-\mathrm{kDa}$ membrane-bound guanosine triphosphate-binding protein involved in growth factor-mediated signal transduction pathways. K-ras is activated through the overexpression or activation of ras-activating signaling partners, including the epidermal growth factor receptor (EGFR) (10). In the present study, the effects of cyclopamine on pancreatic cancer radiosensitivity were investigated in vitro using K-RAS $\mathrm{wt}_{\mathrm{wt}}$ Colo-357 and K-RAS ${ }_{\mathrm{mt}}$ SW-1990 human pancreatic cancer cell lines.

\section{Materials and methods}

Cell culture and reagents. Human pancreatic cancer cell lines, Colo-357 and SW-1990, were purchased from the American 
Type Culture Collection (Manassas, VA, USA). Colo-357 cells were maintained in Dulbecco's modified Eagle's medium and SW-1990 cells were seeded onto tissue culture dishes containing RPMI-1640 medium supplemented with $10 \%$ fetal calf serum, L-glutamine ( $5 \mathrm{mmol} / \mathrm{l})$, non-essential amino acids $(5 \mathrm{mmol} / \mathrm{l})$, penicillin $(100 \mathrm{U} / \mathrm{ml})$ and streptomycin $(100 \mathrm{U} / \mathrm{ml}$; Invitrogen Life Technologies, Carlsbad, CA, USA) at $37^{\circ} \mathrm{C}$ in a humidified $5 \% \mathrm{CO}_{2}$ atmosphere. Cyclopamine and EGF were obtained from Cell Signaling Technology, Inc. (Beverly, MA, USA). The EGFR inhibitor, gefitinib (Iressa), was purchased from AstraZeneca (Macclesfield, UK).

Inhibitor treatment. Stock solutions of the cyclopamine $\mathrm{Hh}$ pathway inhibitor, gefitinib EGFR inhibitor and EGF were prepared at appropriate concentrations in dimethyl sulfoxide (DMSO) and then stored at $-70^{\circ} \mathrm{C}$. For treatment, inhibitor solutions were diluted 1:1,000 to appropriate working concentrations (20 or $40 \mu \mathrm{mol} / 1$ cyclopamine, $10 \mu \mathrm{mol} / 1$ gefitinib and $3 \mu \mathrm{mol} / 1 \mathrm{EGF}$ ) in serum-free medium. Control cultures received medium containing the solvent DMSO at a concentration of $0.1 \%$. Gefitinib and EGF were supplemented to the culture media $0.5 \mathrm{~h}$ before irradiation and $24 \mathrm{~h}$ of preirradiation treatment with cyclopamine was conducted.

Ionizing radiation. A Siemens $6 \mathrm{MV}$ X-ray linear accelerator (Siemens, Munich, Germany) was used to deliver a single dose of ionizing radiation (IR) with a dose rate of $200 \mathrm{cGy} / \mathrm{min}$ at room temperature.

Clonogenic assay. Cells were plated at various cell densities and irradiated with $0.5,1,2,4$ and 6 Gy X-ray $24 \mathrm{~h}$ later. Following 12-14 days incubation at $37^{\circ} \mathrm{C}$, cells were stained with Giemsa. The number of colonies per dish was counted and the surviving fractions were calculated as the ratio of plating efficiencies for irradiated and unirradiated cells. Plating efficiency is defined as the colony number divided by the number of cells plated for unirradiated controls. Experiments were conducted in triplicate and data from three independent experiments are presented as the means $\pm \mathrm{SD}$. All survival fractions were fitted into the linear quadratic model.

Apoptosis assay. Cells were removed with trypsin and collected into centrifuge tubes together with the culture medium. Flow cytometry and Annexin V-fluorescein isothiocyanate (FITC) apoptosis analysis were performed as previously described (11). Cell cycle distribution and apoptotic rate were calculated from $1 \times 10^{-4}$ cells using ModFit LT software with the FACS Calibur (both Becton-Dickinson, San Jose, CA, USA).

Cell cycle assays. Cells were removed with trypsin and collected into centrifuge tubes together with the culture medium. Detailed methods for flow cytometry analysis were previously described (12). Cell cycle distribution was calculated from $1 \times 10^{-4}$ cells using ModFit LT software with the FACS Calibur.

Immunofluorescence assay. Detection of $\gamma$-H2AX foci immunofluorescence was performed to determine residual DNA double-strand breaks (DSBs). Cells grown on coverslips
(Fisher Scientific, Loughborough, UK) were fixed in ice-cold 4\% paraformaldehyde for $30 \mathrm{~min}$, blocked with $3 \%$ bovine serum albumin in phosphate buffer solution (PBS) and then incubated with an antibody against $\gamma-\mathrm{H} 2 \mathrm{AX}$ (ser139; 1:500; Cell Signaling Technology, Inc.) for $2 \mathrm{~h}$ at $4^{\circ} \mathrm{C}$. After washing with PBS, secondary FITC-conjugated antibody was added for $1 \mathrm{~h}$. The slides were washed with PBS and then mounted with mounting medium containing 4',6-diamino-2-phenylindole.

Western blot analysis. Cell lysates were prepared and western blot analysis was performed as previously described (13). Equal aliquots of total cell protein $(50 \mu \mathrm{g} /$ lane $)$ were electrophoresed on sodium dodecyl sulfate-polyacrylamide gels, transferred onto polyvinylidene fluoride membranes and then probed with $\beta$-actin (C-4), DNA-PKcs (G-4), Ku70 (A-9) (Santa Cruz Biotechnology, Santa Cruz, CA, USA; 1:1,000 dilution), $\gamma$-H2AX or p-ATM (Cell Signaling Technology, Inc.; 1:1,000) primary antibodies, followed by horseradish peroxidase-labeled goat anti-mouse (GAM-007) and goat anti-rabbit (SC-2004) IgG secondary antibodies. The protein bands were visualized using an enhanced chemiluminescence system (Union Bioscience Corporation, Hangzhou, China) with prestained markers as molecular size standards. The densitometry of the protein bands was quantified with Quantity One (Bio-Rad, Hercules, CA, USA) and the values were expressed relative to $\beta$-actin (control for loading and transfer). At least three independent experiments were performed for each cell type studied.

Statistical comparisons. Data are presented as the mean \pm SD. Experimental results of the treated and control groups were compared using the two-tailed Student's t-test. All statistical tests were performed using SPSS version 17.0 (SPSS, Inc., Chicago, IL, USA). $\mathrm{P}<0.05$ was considered to indicate a statistically significant difference.

\section{Results}

Cyclopamine enhances radiosensitivity of pancreatic cancer cells. Given that cyclopamine modulates the Hh pathway, the effect of cyclopamine on the radiation clonogenic survival of $\mathrm{K}^{-R} \mathrm{~S}_{\mathrm{wt}}\left(\right.$ Colo-357) and K-RAS $_{\mathrm{mt}}$ (SW-1990) was analyzed in human pancreatic cancer cell lines. The radiosensitizing effect of cyclopamine was confirmed by single-dose irradiation with doses up to $6 \mathrm{~Gy}$. The results revealed that cyclopamine treatment exerted significant radiosensitization of Colo-357 and SW-1990 cells to the clinically relevant radiation dose per fraction of 2 Gy relative to DMSO controls (Fig. 1).

Cyclopamine does not enhance radiation-induced apoptosis. Previous studies have reported induction of apoptosis by cyclopamine (14). Therefore, the rate of apoptosis was examined upon cyclopamine treatment with irradiation compared with DMSO controls. Cyclopamine $(40 \mu \mathrm{mol} / \mathrm{l})$ alone significantly induced apoptosis. However, in combination with irradiation, it failed to induce apoptosis in K-RAS ${ }_{\mathrm{wt}}$ and K-RAS $\mathrm{m}_{\mathrm{mt}}$ pancreatic cancer cells (Fig. 2A).

Cyclopamine treatment does not affect cell cycle redistribution. Cell cycle phases are associated with various degrees of 
A

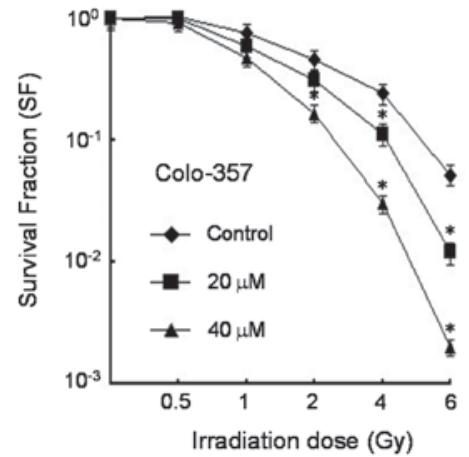

B

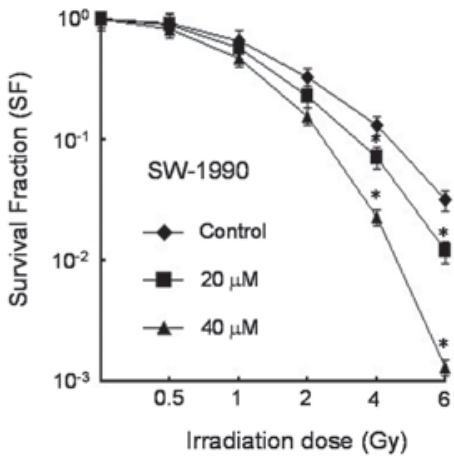

Figure 1. Cyclopamine enhances the radiosensitivity of K-RAS wt $_{\text {t }}$ Colo-357 and K-RAS ${ }_{\mathrm{mt}}$ SW-1990 cells. Log-phase (A) Colo-357 and (B) SW-1990 cells were pretreated for $24 \mathrm{~h}$ with cyclopamine ( 20 or $40 \mu \mathrm{mol} / \mathrm{l}$ ) and cells were plated at various cell densities and irradiated with $0.5,1,2,4$ and $6 \mathrm{~Gy}$ X-ray $24 \mathrm{~h}$ later. Following 14 days, the number of colonies formed was counted and survival fractions were calculated. Data points represent the mean surviving fraction $($ mean \pm SD) of three parallel experiments.
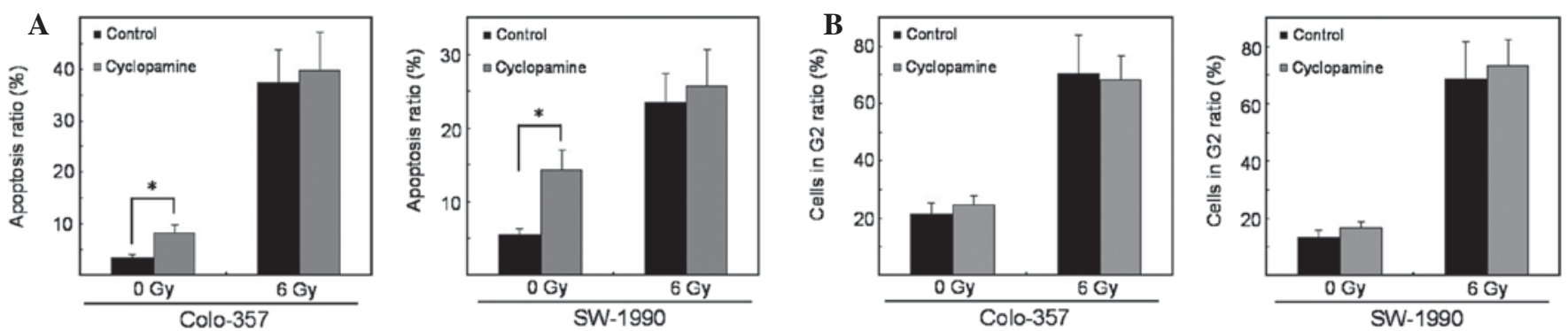

Figure 2. Cyclopamine-treatment does not affect radiation-induced apoptosis and cell cycle redistribution. (A) Following treatment with cyclopamine for $24 \mathrm{~h}$, cells remained unirradiated or received a single dose of $6 \mathrm{~Gy}$. After $48 \mathrm{~h}$, cells were treated as indicated, fixed and then stained with 4',6-diamino-2-phenylindole to microscopically determine cells with typically apoptotic nuclear morphology. (B) Cell cycle distribution was assayed in cells after $24 \mathrm{~h}$ of cyclopamine treatment plus 6 Gy X-rays. The student's t-test was used to compare cyclopamine/irradiated and DMSO/irradiated cells. ${ }^{*} \mathrm{P}<0.05$, vs. control. DMSO, dimethyl sulfoxide.

radiosensitivity. Thus, the percentage of cells in the radiosensitive G2 cell cycle phase was determined upon treatment with cyclopamine alone or in combination with irradiation (15). A significant $\mathrm{G} 2$ cell cycle arrest was noted following irradiation. However, cyclopamine ( $40 \mu \mathrm{mol} / \mathrm{l})$ treatment failed to abrogate radiation-induced G2 arrest as compared with DMSO controls. This effect was observed in K-RAS ${ }_{\mathrm{wt}}$ and K-RAS pancreatic cancer cells (Fig. 2B).

Effects on DNA-DSB repair by cyclopamine. To analyze how cyclopamine affects radiation-induced H2AX phosphorylation as an indicator of DNA damage signaling, Colo-357 and SW-1990 cells were irradiated with a single dose of ionizing radiation (6 Gy). H2AX phosphorylation at Ser139 reached a maximum at $0.5 \mathrm{~h}$ following ionizing radiation (Fig. 3A). Using western blot analysis, a dose-dependent increase in $\gamma$-H2AX following ionizing radiation was observed, particularly at the $0.5 \mathrm{~h}$ time point (Fig. 3B). The formation of $\gamma-\mathrm{H} 2 \mathrm{AX}$ foci was measured $0.5 \mathrm{~h}$ after irradiation of pancreatic cancer cells. This procedure was conducted in order to recognize the molecular mechanisms of cyclopamine radiosensitization. Radiation-induced $\gamma$-H2AX foci were significantly increased in cyclopamine-treated Colo-357 and SW-1990 cells (Fig. 4A), indicative of DNA repair inhibition. Fig. 4B shows the effect of cyclopamine on the expression of DNA repair-related proteins. For the two cell lines, H2AX phosphorylation was enhanced following irradiation. In
A

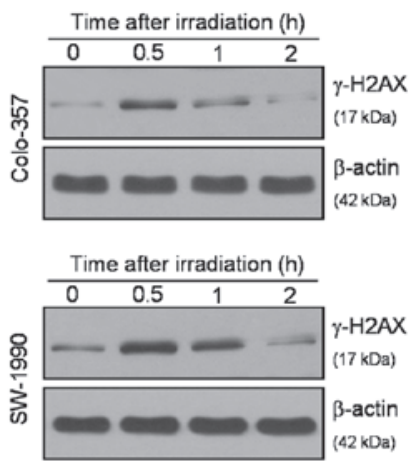

B
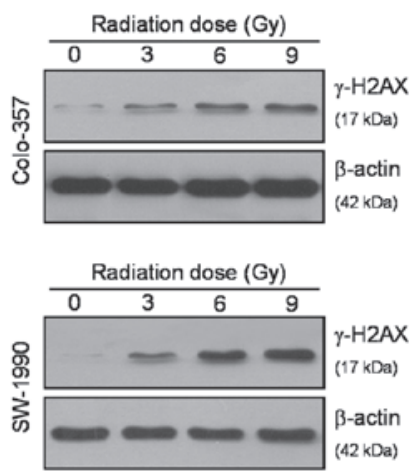

Figure 3. Time kinetics of radiation-induced $\mathrm{H} 2 \mathrm{AX}$ phosphorylation K-RAS wt $_{\mathrm{wt}}$ Colo-357 and K-RAS ${ }_{\mathrm{mt}}$ SW-1990 cells were exposed to ionizing radiation at single doses of 3, 6 or $9 \mathrm{~Gy}$. Cells were lysed at the times indicated, subjected to SDS-PAGE and then incubated with antibodies against $\gamma$-H2AX and $\beta$-actin. 
A

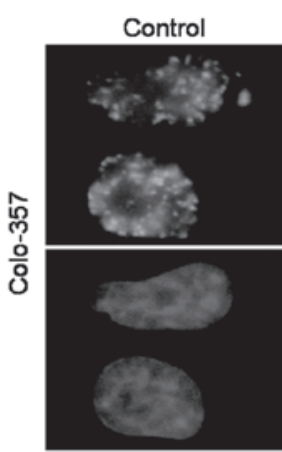

Control

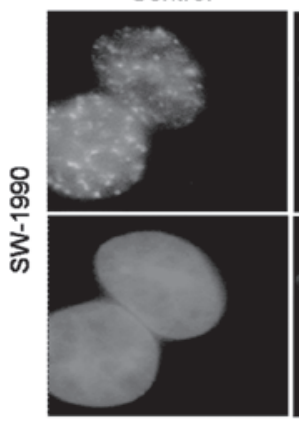

$20 \mu \mathrm{M}$

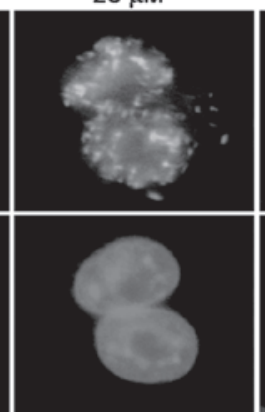

$20 \mu \mathrm{M}$

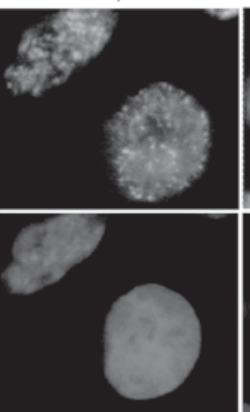

$40 \mu \mathrm{M}$

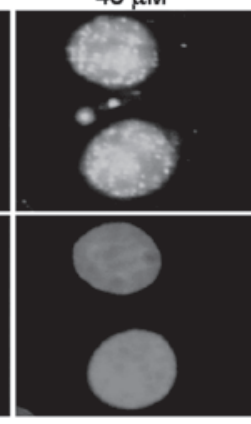

$40 \mu \mathrm{M}$

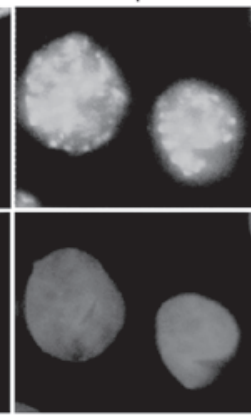

B
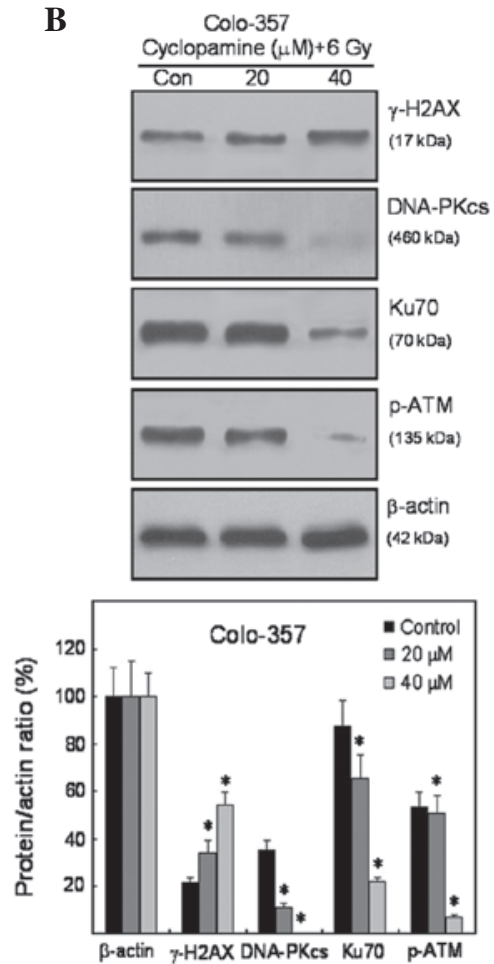

SW-1990
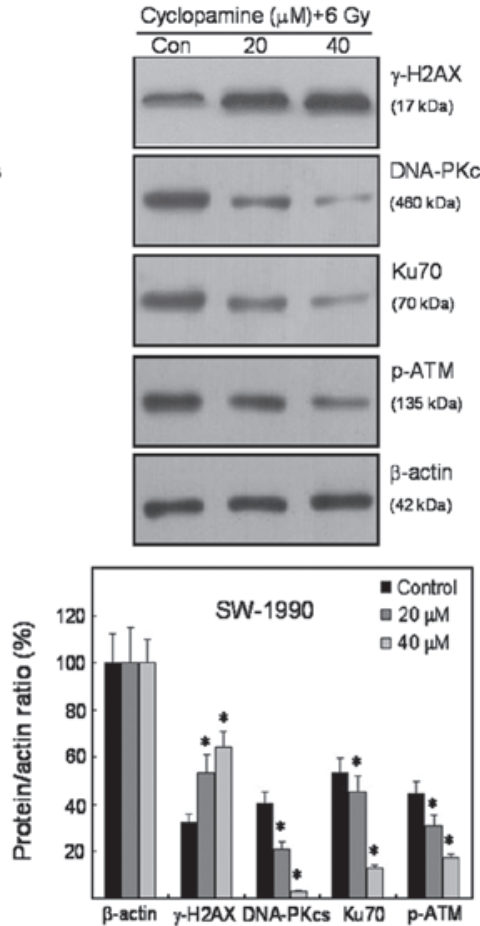

Figure 4. Expression of DNA damage-induced mediator proteins at $0.5 \mathrm{~h}$ after irradiation in Colo-357 and SW-1990 cells following inhibition of the Hh pathway by cyclopamine. (A) Localization of $\gamma-\mathrm{H} 2 \mathrm{AX}$ nuclear foci in single cells was determined $0.5 \mathrm{~h}$ after radiation and cyclopamine administration. Cells treated with cyclopamine were irradiated with a single dose of $6 \mathrm{~Gy}$ X-ray followed by immunofluorescence $0.5 \mathrm{~h}$ later. After cells were fixed, permeabilized, treated with the respective antibodies and stained, color image acquisition was performed by confocal microscopy. (B) Western blot analysis of the expression of $\gamma$-H2AX, DNA-PKcs, Ku70 and p-ATM was performed. Hh, hedgehog.
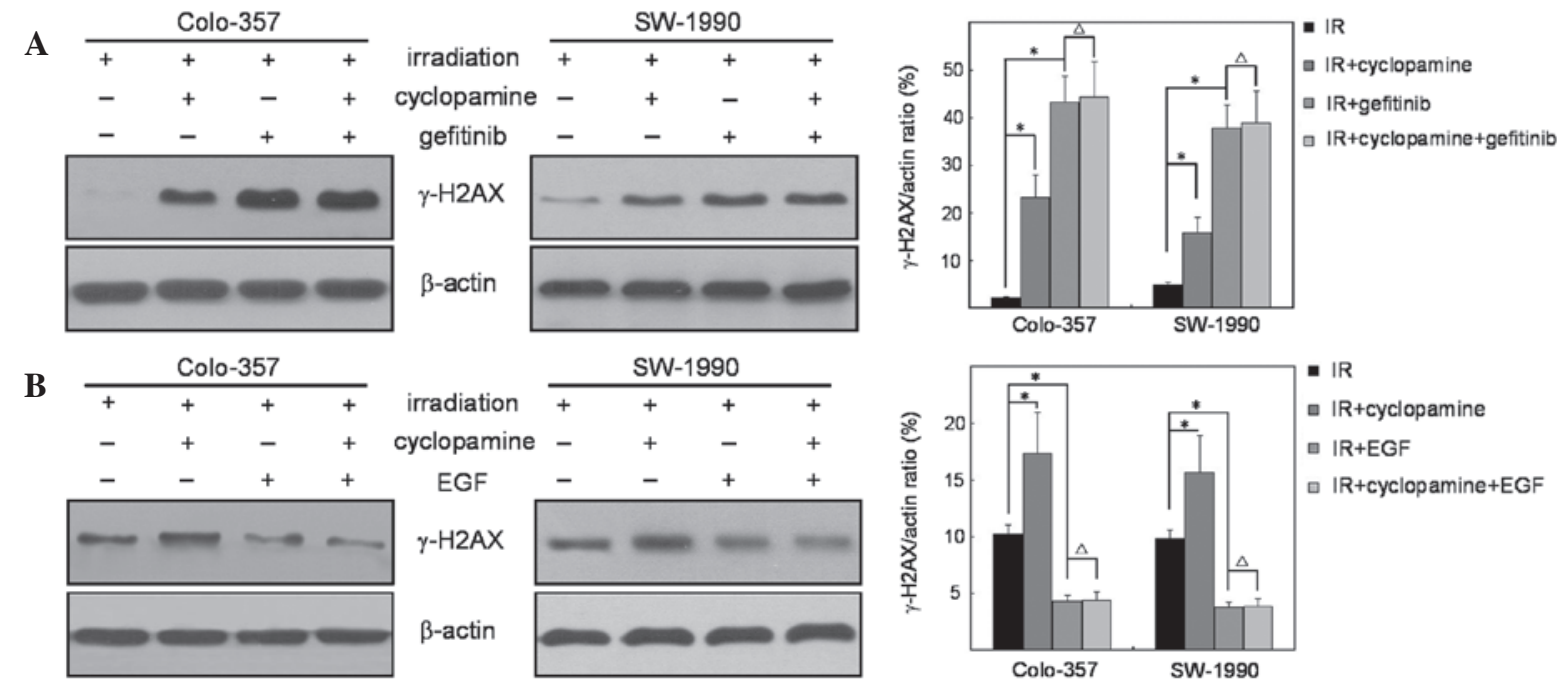

Figure 5. Phosphorylation of H2AX as a function of inhibitor treatment. (A) K-RAS $\mathrm{wt}_{\mathrm{w}}$ Colo-357 and K-RAS $\mathrm{mt}$ SW-1990 cells were treated with cyclopamine ( $40 \mu \mathrm{mol} / \mathrm{l})$ and gefitinib $(10 \mu \mathrm{mol} / \mathrm{l})$ and then exposed to single-dose ionizing radiation (IR) of $6 \mathrm{~Gy}$. (B) Cells were treated with cyclopamine (40 $\mu$ mol/l) and EGF $(3 \mu \mathrm{mol} / 1)$. At $30 \mathrm{~min}$ after ionizing radiation, cells were lysed and lysates were subjected to SDS-PAGE and $\gamma$-H2AX immunoblotting. The densitometry values represent the mean of at least three independent experiments. ${ }^{*} \mathrm{P}<0.05$ and ${ }^{\wedge} \mathrm{P}>0.05$, vs. control group. EGF, epidermal growth factor.

contrast to $\gamma$-H2AX, radiation-induced p-ATM, Ku70 and DNA-PKcs were all inhibited (Fig. 4B).

Cyclopamine inhibits DNA-DSB repair in an EGFR-dependent pathway. To verify the effect of EGFR in cyclopamine-induced radiosensitivity, cells were treated with gefitinib prior to irradiation. The expression levels of $\gamma-\mathrm{H} 2 \mathrm{AX}$ showed that the inhibitory effect of gefitinib blocked cyclopamine-induced H2AX phosphorylation, which is consistent with the results discussed (Fig. 5A). Furthermore, EGF markedly inhibited cyclopamine-induced phosphorylation of $\mathrm{H} 2 \mathrm{AX}$ following irradiation, indicating the dependence of $\mathrm{H} 2 \mathrm{AX}$ phosphorylation partly through an EGFR-dependent pathway (Fig. 5B). 


\section{Discussion}

Pancreatic cancer is the fourth leading cause of cancer-related mortality and is associated with multiple aberrations in cellular signaling cascades (16). The treatment of pancreatic cancer is frequently met with poor outcomes due to the development of resistance to therapy (17). Currently, an effective treatment for pancreatic cancer is lacking and conventional therapy has shown limited success in improving patient survival. Therefore, understanding the mechanisms regulating the molecular changes that drive the refractoriness to therapy is a prerequisite for the development of effective interventions for pancreatic cancer.

In the present study, the effect of cyclopamine on the radiation clonogenic survival of pancreatic cancer cell lines was analyzed. Cyclopamine treatment exerted significant radiosensitization on $\mathrm{K}-\mathrm{RAS}_{\mathrm{wt}}$ and $\mathrm{K}-\mathrm{RAS}_{\mathrm{mt}}$ pancreatic cancer cell lines, indicating cyclopamine-induced radiosensitivity through a K-RAS-independent pathway. Apoptosis and cell cycle assays showed that cyclopamine failed to affect radiation-induced apoptosis and cell cycle redistribution. In addition, phospho-H2AX was analyzed following ionizing radiation to determine the underlying mechanisms. Radiation-induced $\gamma$-H2AX foci were significantly increased in cyclopamine-treated cells. Western blot analysis showed that radiation-induced p-ATM, Ku70 and DNA-PKcs were all inhibited in cyclopamine-treated cells. To verify the underlying mechanisms in cyclopamine-induced DNA-DSB repair, cells were treated with gefitinib or EGF prior to irradiation. The results indicated that the cyclopamine-induced activity of H2AX occurred, in part, through an EGFR-dependent pathway.

The results of the present study provide convincing evidence for the function of inhibited Hh pathway in pancreatic cancer. This study may serve as a basis for clinical studies identifying the role of cyclopamine in pancreatic cancer radiotherapy. In conclusion, these observations indicate that the role of cyclopamine in the radiosensitivity of pancreatic cancer may be important for translational research on the development of more effective and targeted therapeutic strategies for pancreatic cancer.

\section{Acknowledgements}

This study was supported by grants from the Hospital Center Technology Development Fund of Wuxi City (no. YGM1101) and the Social Development Project of Kunshan City (no. KS1224).

\section{References}

1. Maitra A and Hruban RH: Pancreatic Cancer. Annu Rev Pathol 3: 157-188, 2008.

2. Zeng H, Yu H, Lu L, et al: Genetic effects and modifiers of radiotherapy and chemotherapy on survival in pancreatic cancer. Pancreas 40: 657-663, 2011.

3. Stan SD, Singh SV and Brand RE: Chemoprevention strategies for pancreatic cancer. Nat Rev Gastroenterol Hepatol 7: 347-356, 2010.

4. Mahindroo N, Punchihewa C and Fujii N: Hedgehog-Gli signaling pathway inhibitors as anticancer agents. J Med Chem 52: 3829-3845, 2009.

5. Mazumdar T, Devecchio J, Agyeman A, et al: Blocking hedgehog survival signaling at the level of the GLI genes induces DNA damage and extensive cell death in human colon carcinoma cells. Cancer Res 71: 5904-5914, 2011.

6. Feldmann G, Dhara S, Fendrich V, et al: Blockade of hedgehog signaling inhibits pancreatic cancer invasion and metastases: a new paradigm for combination therapy in solid cancers. Cancer Res 67: 2187-2196, 2007.

7. Kumar SK, Roy I, Anchoori RK, et al: Targeted inhibition of hedgehog signaling by cyclopamine prodrugs for advanced prostate cancer. Bioorg Med Chem 16: 2764-2768, 2008.

8. Bartsch DK, Sina-Frey M, Lang S, et al: CDKN2A germline mutations in familial pancreatic cancer. Ann Surg 236: 730-737, 2002.

9. Almoguera C, Shibata D, Forrester K, et al: Most human carcinomas of the exocrine pancreas contain mutant c-K-ras genes. Cell 53: 549-554, 1988.

10. Toulany M, Kasten-Pisula U, Brammer I, et al: Blockage of epidermal growth factor receptor-phosphatidylinositol 3-kinaseAKT signaling increases radiosensitivity of K-RAS mutated human tumor cells in vitro by affecting DNA repair. Clin Cancer Res 12: 4119-4126, 2006.

11. Jiao Y, Wang HC and Fan SJ: Growth suppression and radiosensitivity increase by HMGB1 in breast cancer. Acta Pharmacol Sin 28: 1957-1967, 2007.

12. Jiao Y, Ge CM, Meng QH, et al: Adenovirus-mediated expression of Tobl sensitizes breast cancer cells to ionizing radiation. Acta Pharmacol Sin 28: 1628-1636, 2007.

13. Jiao Y, Sun KK, Zhao L, et al: Suppression of human lung cancer cell proliferation and metastasis in vitro by the transducer of ErbB-2.1 (TOB1). Acta Pharmacol Sin 33: 250-260, 2012.

14. Che J, Zhang FZ, Zhao CQ, et al: Cyclopamine is a novel Hedgehog signaling inhibitor with significant anti-proliferative, anti-invasive and anti-estrogenic potency in human breast cancer cells. Oncol Lett 5: 1417-1421, 2013.

15. Ahsan A, Hiniker SM, Davis MA, et al: The role of cell cycle in epidermal growth factor receptor inhibitor-mediated radiosensitization. Cancer Res 69: 5108-5114, 2009.

16. Cloos CR, Daniels DH, Kalen A, et al: Mitochondrial DNA depletion induces radioresistance by suppressing G2 checkpoint activation in human pancreatic cancer cells. Radiat Res 171: 581-587, 2009.

17. Kimple RJ, Vaseva AV, Cox AD, et al: Radiosensitization of EGFR/HER2 positive pancreatic cancer is mediated by inhibition of Akt independent of Ras mutational status. Clin Cancer Res 16: 912-923, 2010. 